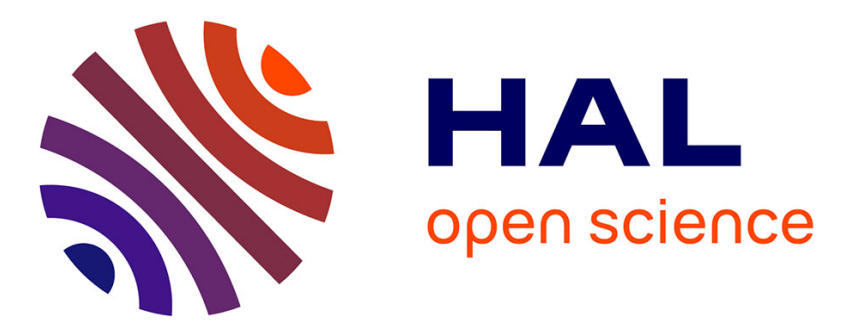

\title{
Correlation Function Analysis of Geomagnetic Field Reversals
}

\author{
C. Laj, D. Nordemann, Y. Pomeau
}

\section{To cite this version:}

C. Laj, D. Nordemann, Y. Pomeau. Correlation Function Analysis of Geomagnetic Field Reversals. Journal of Geophysical Research, 1979, 84 (B9), pp.4511. 10.1029/JB084iB09p04511 . hal-03408861

\section{HAL Id: hal-03408861 https://hal.science/hal-03408861}

Submitted on 29 Oct 2021

HAL is a multi-disciplinary open access archive for the deposit and dissemination of scientific research documents, whether they are published or not. The documents may come from teaching and research institutions in France or abroad, or from public or private research centers.
L'archive ouverte pluridisciplinaire HAL, est destinée au dépôt et à la diffusion de documents scientifiques de niveau recherche, publiés ou non, émanant des établissements d'enseignement et de recherche français ou étrangers, des laboratoires publics ou privés. 


\title{
Correlation Function Analysis of Geomagnetic Field Reversals
}

\author{
C. LaJ and D. NordemanN \\ Centre des Faibles Radioactivités, Laboratoire Mixte CNRS-CEA, 91190 Gif-sur-Yvette, France
}

\section{Y. Pomeau}

\author{
Service de Physique Théorique, Centre d'Etudes Nucléaires de Saclay, 91190 Gif-sur-Yvette, France
}

\begin{abstract}
We have analyzed the time series of geomagnetic field reversals using a correlation function of the telegraphic signal obtained from the Heirtzler scale by assigning \pm 1 to intervals of normal/reversed polarity. Unlike the correlation function used by Naidu, the autocorrelation function we define is only very slightly sensitive to undetected short polarity intervals. Thus the results we obtain are valid irrespective of failure to detect short polarity intervals and give information about the dynamics of the time series of polarity reversals. We show that, within statistical noise limits, the successive polarity intervals are statistically independent and are distributed in time according to Poisson process, as already suggested by Cox using other mathematical means.
\end{abstract}

\section{INTRODUCTION}

There have been a number of articles about the statistics of geomagnetic reversals. As initially recognized by Cox [1968], one of the difficulties to overcome when doing such statistics is the extreme sensitivity of the histogram of polarity events to the proportion of short polarity events. Indeed, Cox [1968] has shown how such a histogram completely changed as the discovery of polarity events modified the first quantitative time scale which appeared in 1963 when only polarity epochs were known. This sensitivity of course is due to the fact that the earth's geomagnetic field has only two polarity states; thus a short polarity event inserted in the middle of a long period of opposite polarity not only adds the short polarity event to the histogram but also rubs out a long period and adds the two corresponding middle-length periods.

Cox [1968] has proposed a basic model in which geomagnetic reversals occur as a result of an interaction of long-period oscillations of the geomagnetic dipole field and stochastic variations of the nondipole field which act as a triggering mechanism for reversals. Other models have also since been proposed, some involving cyclonic convection cells [Parker, 1969; Levy, 1972a,b,c] or related mechanisms [Nagata, 1969; Cox, 1970, 1975]. We shall mostly discuss the model of Cox [1968] in this paper.

This model predicts that geomagnetic reversals are Poisson distributed in length. While there is a very statisfactory overall agreement between the predictions of the model and the observed rate, a discrepancy is observed for the short polarity events; i.e., the model predicts more short polarity events than are observed, especially in the ages between 55 and 75 m.y. Since a short polarity event can easily be undetected by our means of detection, a great deal of work has been done in order to attain the real rate of reversals. Indeed there is evidence of many previously undetected short polarity events [Cox, 1969; Harrison, 1969; Blakely and Cox, 1972; Tacier et al., 1975].

In this paper we have chosen another way of reasoning. Instead of using mathematical methods to attain the true rate of reversal, we have tried to define a statistical mathematical quantity which is by its nature not too sensitive to short polarity events. The autocorrelation function of the 'telegraphic signal' made of the sign of the axial component of the

Copyright (C) 1979 by the American Geophysical Union. geomagnetic field, such as it is defined in the next paragraph, is such a quantity. Naidu $[1971,1975]$ has also already attempted an approach using correlation functions. However, the autocorrelation function he uses is by definition sensitive to short polarity events and thus has the same drawbacks as mentioned above. Also Phillips and Cox [1976] have performed spectral analysis of this same telegraphic signal.

While a quantity which is unsensitive to the high-frequency part of the spectrum is in itself limited and cannot give the same fine results as the analysis of Cox and co-workers, we feel that the dynamical nature of the autocorrelation function gives new results as shown further on. In particular, we shall show that other models are compatible with the observed results, which do not need any triggering mechanism as in Cox's model. This is important, since it is very difficult to trigger a long-period oscillator using short-period stochastic fluctuation of reasonable amplitude because of general vibration mechanics reasons. This point is probably a weakness in Cox's and related models, as discussed below.

\section{Mathematical Methods}

Our autocorrelation function is defined as follows: first we attribute the sign +1 to normal polarity intervals and -1 to inverse polarity intervals. Then we construct the telegraphic signal, which gives the polarity scale. (The Heirtzler time scale is used throughout this paper.) We then consider this signal as being random and stationary. This last assumption might be unjustified, because it is possible that the dynamics of reversals has changed over geological times. On the other hand, we believe that it is difficult, if not impossible, to conclude from the data that the process of geomagnetic reversals is nonstationary.

The autocorrelation function is then defined in the usual way:

$$
C(\tau)=\langle F(t) F(t+\tau)\rangle
$$

where $F(t)= \pm 1$ and the angle brackets indicate a time running average. As shown in Figure 1 for a particular value of $\tau$, $C(\tau)$ is a quantity proportional to the hatched area minus the dotted area. It is easily seen that the addition of a short polarity interval will not significantly affect its value, such an interval contributing a negligible area.

As mentioned earlier, another autocorrelation function may 


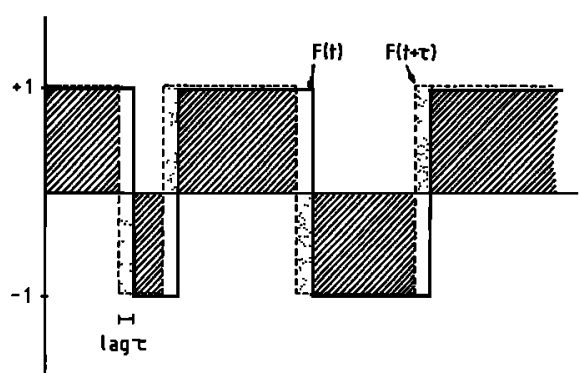

Fig. 1. Schematic representation of how the autocorrelation function is obtained. From the definition $C(\tau)=(1 / T) \int_{0}^{T} F(t) F(t+\tau) d \tau$ it follows that for a particular value of $\tau, C(\tau)$ is proportional to the hatched areas $(F(t)$ and $F(t+\tau)$ of the same sign) minus the dotted areas $(F(t)$ and $F(t+\tau)$ of the opposite sign ).

be defined. In a previous work, Naidu [1975] has considered the correlation function of the length of the various polarity intervals. He defines a correlation function which is sensitive again to short polarity events.

Assuming that the various intervals are chosen at random with a given probability distribution, it is possible [Lee, 1960] to relate in a well-defined way the statistics of the lengths of the intervals and the previously defined autocorrelation function $C(\tau)$. It is worthwhile to notice that the histogram of the lengths of the intervals gives rather indirect information about the underlying dynamical process. This is because the correlation function is a dynamical quantity, while the histogram is of a static nature. When looking at a histogram, one knows that an interval of length, say, $\Delta_{l}$, has occurred $i$ times, but nothing is known about such things as, What was the length of the interval preceding it or, Has a short period more chances to occur after a long one or not?

The autocorrelation function on the other hand is sensitive to the time series of polarity intervals and contains in fact complete information about the time spectrum of the function analyzed. In particular, it indicates whether or not the reversals follow a random process.

If the different polarity intervals considered in the Heirtzler scale were completely random, one should obtain for the autocorrelation function a function decreasing to zero over a time scale of the order of the mean duration of an interval. Let us call this the 'static correlation' width. Then in a naive way one may say that if the correlation function has a width appreciably larger than the static correlation width, then the polarity intervals are dynamically correlated, i.e., the mechanism which causes them possesses a memory. (An extreme case is the one corresponding to the existence of an underlying periodic or eventually multiperiodic mechanism, for which the memory extends over infinite time. In this case the width of the selfcorrelation function becomes ill-defined because the function does not decrease to zero for large time intervals.)

\section{RESULTS}

The autocorrelation function obtained using all the experimental points of the Heirtzler scale is shown in Figure 2. Some theoretical points corresponding to the static correlation function of a sample of 172 polarity reversals are also shown in the figure, as well as the statistical noise (at $95 \%$ confidence level) corresponding to such a sample; these quantities are calculated using the results of Lee [1960] and those of the appendix.

It is evident from the figure that the correlation function is quite similar to the one corresponding to a static correlation, i.e., no dynamical correlation at all. This result is confirmed by a more refined mathematical fit which shows that the observed autocorrelation function is almost identical with the one generated by a Poisson's process. This result disagrees with the original observation of Naidu [1975], whose correlation function showed a width greater than that of the static correlation. In a following paper, Naidu [1976] has shown that most of this width is contributed by the reversals in the 48- to 72-m.y. period. He then divides the entire time span into two periods: one 0-48 m.y., for which the reversal sequence is uncorrelated, and the other 48-72 m.y., for which the sequence is correlated. This separation corresponds to a change in the Heirtzler scale, the mean duration of the interval being greater in the period 48-72 m.y. than in the period 0-48 m.y. Of course, this will cause an increase in the static correlation width which, as mentioned above, is of the order of the mean duration of the polarity intervals. We feel that this effect and not a correlated sequence causes the large width of Naidu's correlation function in the period 48-72 m.y.

In any case we do find an effect of the 48- to 72-m.y. period, but it is not significant when the finiteness of the sample is taken into account. We also have time-compressed the intervals of this period in order to obtain the same mean duration. This is because the question of the stationarity of the random signal has been raised [Ulrych and Clayton, 1976]. Again the autocorrelation function is only slightly affected. We therefore believe the entire sequence of polarity intervals to be uncorrelated, at least at the level of accuracy inherent to the finite sampling method used.

While this result confirms the results already obtained by Cox and co-workers, it is important to note that our result has been obtained on a dynamic and not statical basis as in the case of Cox. In fact, we would here like to discuss a point which seems to us to be a weakness in Cox's as well as other statistical models.

First of all, let us remark that there exists a wide class of systems of ordinary differential equations, designed to represent forced dissipative hydrodynamical systems such as geodynamos, which have nonperiodic solutions. Such systems may oscillate randomly between two states or fixed points; i.e., they may have two unstable polarity states [Lorenz, 1963]. The well-known Rikitake double-disk model belongs to such a

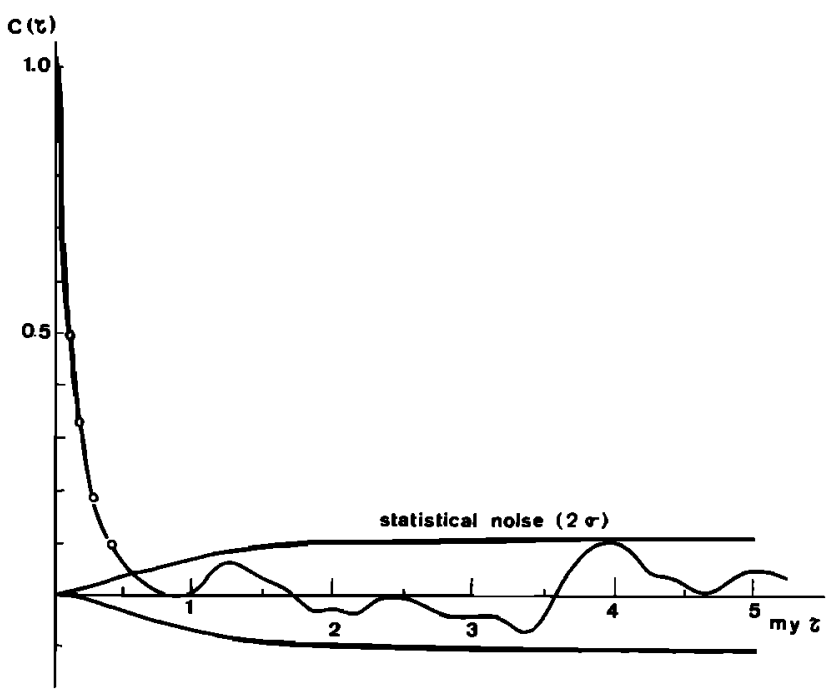

Fig. 2. The autocorrelation function obtained from the Heirtzler time scale. Open circles correspond to a static correlation. The statistical noise level is also indicated. 


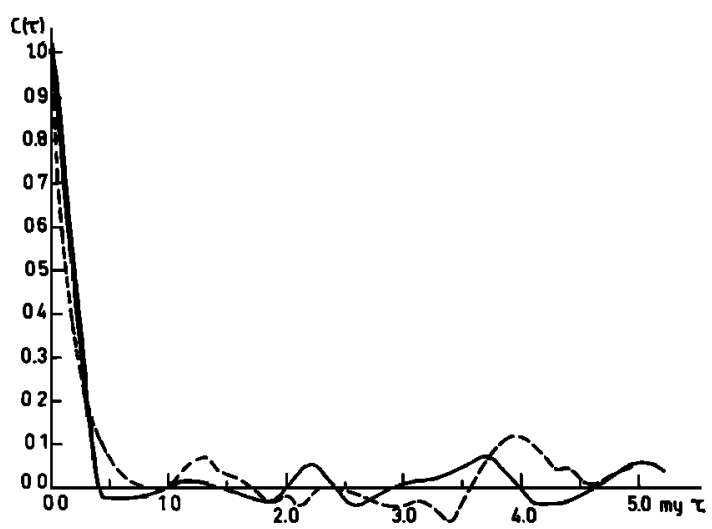

Fig. 3. The autocorrelation function for the Lorenz system (solid line) compared to the one for the Heirtzler scale (dashed line).

class, and indeed such a model displays nonperiodic reversals in the absence of any triggering mechanism whatsoever.

We have calculated the autocorrelation function of one such system, the set of original Lorenz equations [Lorenz, 1963], shown in Figure 3, and found that the self-correlation function is very similar to that observed for the earth's magnetic field. Thus many deterministic nontriggered systems can give rise to observed Poisson-like statistics at least within the accuracy corresponding to finite sampling.

In Cox's original model, random fluctuations of the nondipole field are used to trigger the steady oscillations of the geomagnetic field into a different polarity. Of course, many physical processes are known where triggering effects are important. Nevertheless, we feel that in this case there is a mechanical difficulty arising from the different time constants of the two phenomena. Oscillations of the dipole field typically have a time constant of $2 \times 10^{4}$ years [Cox, 1969]. Characteristic times for the nondipole field range up to about $10^{\mathrm{s}}$ years; however, most of the power is concentrated in the $2 \times 10^{2}$ to $4 \times 10^{2}$ year region. It is known in vibration mechanics that it is quite difficult to couple two systems with very different time constants (for example, it is easier to drive on a bumpy road at a relatively high speed because the car suspension (with a lowfrequency resonance) will not couple with the high-frequency bumps, and the ride will be smoother than at lower speed).

In Cox's model the condition for a polarity change was that the quantity $M_{A}+M_{A}^{\prime}$ change sign (where $M_{A}$ and $M_{A}^{\prime}$ are

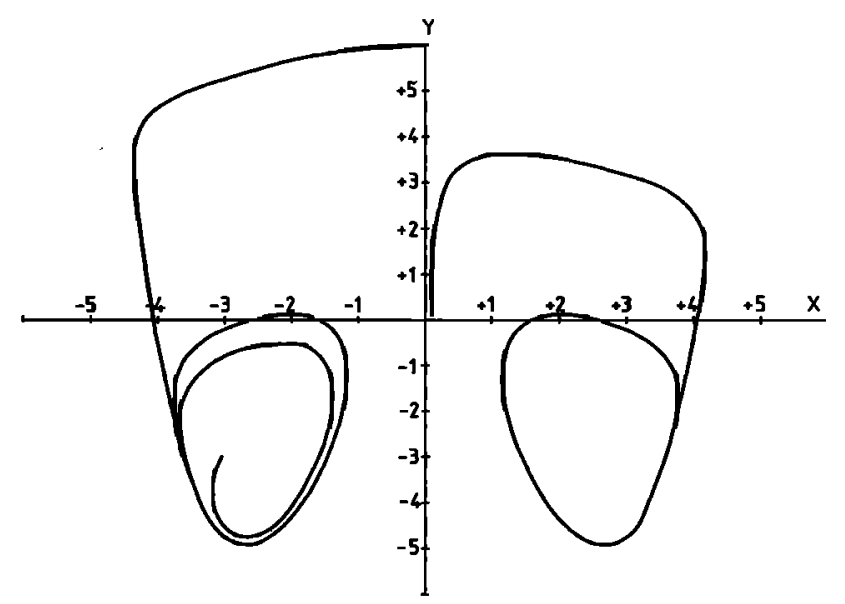

Fig. 4a. Parametric representation of the differential system $\dot{x}=x y$ $+x^{s}+b x^{6} ; \dot{y}=m\left(y+s-e x^{2}\right)$ showing the two limiting cycles about which the system will oscillate ( $b, m, s$, and $e$ being constants). the axial components of the dipole and nondipole fields, respectively). Of course, if this quantity changes sign, there will be a geomagnetic reversal. However, our point is that this rapid fluctuation will not be 'dynamo sustained' but only have a very short lifetime, of the order of the time constant of the nondipole field.

As an example of this behavior, we have devised a system which may oscillate about the two polarity states shown in Figure 4. This system differs from the Lorenz system because there will be no spontaneous transition from one limiting cycle to the other. Rather, as shown in Figure 4, where solutions of this system are identified with trajectories in the phase space, if the initial conditions place the moving point in the $X>0$ region, the system will oscillate indefinitely about the ' $A$ ' state (Figure $4 a$ ). Starting on the contrary in the half-plane $X<0$, it will oscillate about the 'B' state (Figure $4 b$ ). Now let us put the system about the $A$ state and apply a short but intense perturbation to make the representative moving point jump into the $X<0$ zone. The perturbation time length is about $\frac{1}{6 t}$ of the duration of a cycle, i.e., a ratio comparable to the one observed in the case of the dipole and nondipole fields. It can be seen that the system does not begin to oscillate about the B state, but comes back spontaneously to the $X>0$ region to oscillate around the A state after a short transition time. Only an unrealistically strong perturbation would make it jump. This, of course, is contrary to what one would expect on the basis of Cox's model and is the reason why we feel that this model is very difficult to treat dynamically.

The problem of the difference between the time constants of the dipole and nondipole fields is also apparent in other models. Parker himself points out that the quantitative nature of the cyclones is not known well enough to calculate the minimum amount of time during which they have to be absent from low latitudes to induce a reversal. This is, of course, because when cyclones migrate in the high latitudes, only the growth (or decay) rate of the geomagnetic field will reverse sign, not the field itself. His own calculations lead him to the conclusion that reversals as often as $10^{5}$ years are not to be expected. This might lead to a difficulty if, as it appears, many short polarity events have existed.

Lorenz's model has initially been introduced in connection with meteorological studies. Later, Robbins [1975] has given a set of ordinary differential equations for the dynamics of a selfsustained dynamo and shown that both Rikitake's double-disk

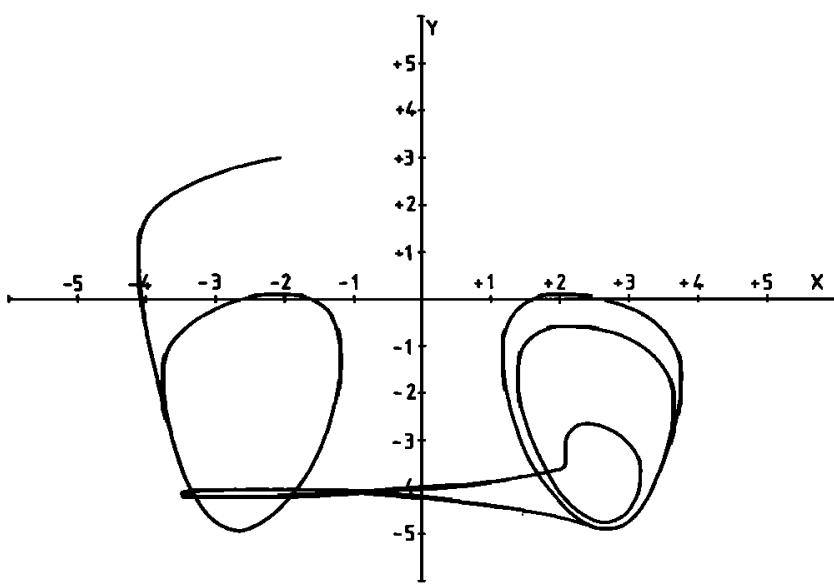

Fig. 4b. Same representation with an additional pulse which makes the system jump from the $x>0$ to the $x<0$ region. As it is seen, the point representative of the system comes back naturally in the $x>0$ region. 
dynamo and Lorenz's model are particular cases of this system. In all cases, solutions have been obtained numerically, leading to a rather complicated behavior. Recently, however, Nozières [1978] has remarked that the existence of two welldefined and quite different time constants for the geodynamo $\tau_{1}$ and $\tau_{2}$ representing the time necessary to dump an electric current and the time constant of exchanges between kinetic and magnetic energies, respectively, allows the problem to be solved analytically $\left(\tau_{1}\right.$ is of the order of $10^{4}-10^{5}$ years, and $\tau_{2}$ of the order of $1-100$ years, so that $\tau_{2} \ll \tau_{1}$ ).

Nozières has solved a very simple model and shown that in some realistic conditions the system will be in a "released relaxation' state, undergoing periodical reversals at intervals of time $\tau_{1}$, while the length of a single reversal is $\tau_{2}$. Addition of more electromagnetical degrees of freedom destroys the periodicity in the reversal frequency, thus leading to a behavior similar to the earth's magnetic field. The analytical solution of Nozières clearly shows physical reasons of the nonperiodicity and suddenness of the reversals, and we have shown that the autocorrelation function of such a system is similar to the one computed for the earth's field. While a complete solution is out of view for the moment, we think that consideration of the Lorenz model may lead to a better understanding of and maybe to selecting those patterns of convection and forces which could give rise to spontaneous reversals of the geomagnetic field.

ApPEndix: Evaluation of the Statistical Noise OF THE A UTOCORRELATION FunCtion AsSOCIATED With Finite SAMPLING OF UNCORRELATEd R EVERSALS

The quantity to be calculated is

$$
\begin{aligned}
\left\langle\delta C^{2}(t)\right\rangle= & \frac{1}{(T-t)^{2}} \int_{\tau}^{\tau+T-t} d t_{1} \int_{\tau}^{\tau+T-t} d t_{2} \\
& \cdot\left[\left\langle\sigma\left(t_{1}\right) \sigma\left(t_{1}+t\right) \sigma\left(t_{2}\right) \sigma\left(t_{2}+t\right)\right\rangle-\langle C(t)\rangle^{2}\right]
\end{aligned}
$$

where $\sigma(t)$ is the value at time $t$ of a Poisson rectangular wave; i.e., $\sigma(t)= \pm 1$ and $C(t)=\exp \left(-2|t| / \tau_{0}\right)$ is the autocorrelation function of this wave [Lee, 1960].

The four-term correlation function appearing in (1) is difficult to calculate. It is, however, possible to reduce it to a usual two-term correlation function in the following way [ $\mathrm{Van} \mathrm{Kam-}$ pen. 1976]. Let $P\left(a, t_{a} ; b, t_{b}\right)$ be the probability that the Poisson wave is in the $a$ state at time $t_{a}$ and in the $b$ state at time $t_{b}$ ( $a$ and $b$ can only have the values plus or minus). To calculate the two-time autocorrelation function, one has to consider four functions, depending only on $t_{0}-t_{a}=\theta(\theta>0)$, which are

$$
\begin{array}{ll}
P(+, 0 ;+, \theta) & P(+, 0 ;-, \theta) \\
P(-, 0 ;+, \theta) & P(-, 0 ;-, \theta)
\end{array}
$$

These functions are related by a system of four linear differential equations of first order of the form

$$
\frac{d}{d \theta} P(+, 0 ;+, \theta)=\frac{1}{t_{0}}[P(+, 0 ;-, \theta)-P(+, 0 ;+, \theta)]
$$

where $1 / \tau_{0}$ is the probability of a polarity change per unit time. The three other differential equations are analogous.

Using these probability functions, the two-time autocorrelation function becomes

$$
\begin{aligned}
\left\langle\sigma\left(t_{1}\right) \sigma\left(t_{2}\right)\right\rangle= & P\left(+, t_{1} ;+, t_{2}\right)+P\left(-, t_{1} ;-, t_{2}\right) \\
& -P\left(-, t_{1} ;+, t_{2}\right)-P\left(+, t_{1} ;-, t_{2}\right) \quad t_{2} \geq t_{1}
\end{aligned}
$$

The probability functions $\boldsymbol{P}$ obey the evolution equations (2) and must furthermore meet the limiting conditions

$$
\begin{aligned}
& P\left(+, t_{1} ;+, t_{1}\right)=P\left(-, t_{1} ;-, t_{1}\right)=\frac{1}{2} \\
& P\left(+, t_{1} ;-, t_{1}\right)=P\left(-, t_{1} ;+, t_{1}\right)=0
\end{aligned}
$$

After resolution, one finds, of course,

$$
\left\langle\sigma\left(t_{1}\right) \sigma\left(t_{2}\right)\right\rangle=\exp \left(-2\left|t_{2}-t_{1}\right| / t_{0}\right) \quad t_{2} \geq t_{1}
$$

A simple generalization of this calculation allows the calculation of the four-term correlation function appearing in (1). Indeed, we have

$$
\begin{aligned}
& \left\langle\sigma\left(t_{a}\right) \sigma\left(t_{b}\right) \sigma\left(t_{c}\right) \sigma\left(t_{d}\right)\right\rangle=\exp \left[-2\left(t_{d}-t_{c}\right) / t_{0}\right] \\
& \cdot \exp \left[-2\left(t_{b}-t_{a}\right) / t_{0}\right] \quad t_{d} \geq t_{c} \geq t_{b} \geq t_{a}
\end{aligned}
$$

One may then rewrite expression (1) in the form

$$
\begin{aligned}
\left\langle\delta C^{2}(t)\right\rangle & =\frac{2}{(T-t)^{2}} \int_{0}^{T-t} d t_{1} \int_{0}^{t_{2}} d t_{2} \\
\cdot & {\left[\left\langle\sigma\left(t_{1}\right) \sigma\left(t_{1}+t\right) \sigma\left(t_{2}\right) \sigma\left(t_{2}+t\right)\right\rangle-\exp \left(-2|t| / t_{0}\right)\right] }
\end{aligned}
$$

Then (3) shows that the integrand in (4) is zero when $t_{1}+t \geq t_{2}$ and that otherwise, one has

$$
\begin{aligned}
\left\langle\delta C^{2}(t)\right\rangle= & \frac{2}{(T-t)^{2}} \int_{0}^{T-t} d t_{1} \int_{t_{1}-t}^{t_{1}} d t_{2}\left[\exp \left(-2\left|t_{1}-t_{2}\right| / t_{0}\right)\right. \\
& \left.-\exp \left(-2|t| / t_{0}\right)\right] \\
= & \frac{t_{0}}{(T-t)} \phi\left(t / t_{0}\right)=\langle\delta C(\infty)) \phi\left(t / t_{0}\right)
\end{aligned}
$$

where $\phi(x)=1-\exp (-x)-x \exp (-x)$.

In the limit $x \rightarrow 0$, this expression becomes

$$
\underset{x \rightarrow 0}{\phi(x)} \cong \frac{x^{2}}{2}+O\left(x^{3}\right)+\cdots
$$

Thus $\left\langle\delta C^{2}(t)\right\rangle$ is zero when $t \rightarrow 0$, which is what we expect because for $t=0$ the value $C(0)=1$ is perfectly determined. When $t \rightarrow \infty, \phi(x)=1$; and $\delta^{2} C(\infty)$, substituting $N=(T-t) /$ $t_{0}$ (number of reversals in the period $T-t$ ), becomes

$$
\left(\delta^{2} C(\infty)\right)=1 / 2 N
$$

It should be noted that this is a theoretical value and thus differs in essence from the estimation of noise level of Phillips and Cox, which is an estimation a posteriori using a $\chi^{2}$ test on the observed results.

\section{REFERENCES}

Blakely, R. J., and A. Cox, Evidence for short geomagnetic polarity intervals in the early Cenozoic, J. Geophys. Res., 77, 7065-7072, 1972.

Cox, A., Lengths of geomagnetic polarity intervals, J. Geophys. Res., 73, 3247-3260, 1968.

Cox, A., Geomagnetic reversals, Science, 163, 237-245, 1969.

Cox, A., Reconciliation of statistical models for reversals, J. Geophys. Res., 75, 7501-7503, 1970.

Cox, A., The frequency of geomagnetic reversals and the symmetry of the nondipole field, Rev. Geophys. Space Phys., 13, 35-51, 1975.

Harrison, C. G. A., What is the true rate of reversals of the Earth's magnetic field?, Earth Planet. Sci. Lett., 6, 186-188, 1969.

Lee, Y. W., Statistical Theory of Communication, 53 pp., John Wiley, New York, 1960.

Levy, E., Effectiveness of cyclonic convection for producing the geomagnetic field, Astrophys. J., 17I, 62I-633, $1972 a$. 
Levy, E., Kinematic reversal schemes for the geomagnetic dipole, Astrophys. J., 171, 635-642, $1972 b$.

Levy, E., On the state of the geomagnetic field and its reversals, Astrophys. J., I75, 573-581, 1972c.

Lorenz, E. N., Deterministic non-periodic flow, J. Atmos. Sci., 20, 130-141, 1963.

Nagata, T., Length of geomagnetic polarity intervals, J. Geomagn. Geoelec. 21, 701-704, 1969.

Naidu, P. S., Statistical structure of geomagnetic field reversals, $J$. Geophys. Res., 76, 2649-2662, 1971.

Naidu, P. S., Second-order statistical structure of geomagnetic field reversals, J. Geophys. Res., 80, 803-806, 1975.

Naidu, P. S., Reply, J. Geophys. Res., 81, 1034, 1976.

Nozières, $P$., Les retournements du champ magnétique terrestre: Quelques spéculations, Bull. Soc. Fr. Phys., 30, 2-6, 1978.

Parker, E. N., The occasional reversal of the geomagnetic field, $A s$ trophys. J., 158, 815-827, 1969.
Phillips, J., and A. Cox, Spectral analysis of geomagnetic reversal time scales, Geophys. J. Roy. Astron. Soc., 45, 19-33, 1976.

Robbins, K. A., Disk dynamos and magnetic reversal, Ph.D. thesis, Mass. Inst. of Technol., Cambridge, Mass., 1975.

Tacier, J.-D., P. Switzer, and A. Cox, A model relating undetected geomagnetic polarity intervals to the observed rate of reversals, $J$. Geophys. Res., 80, 4446-4448, 1975.

Ulrych, T. J., and R. W. Clayton, Comment on 'Second-order statistical structure of geomagnetic field reversals' by P. S. Naidu, $J$. Geophys. Res., 81, 1033, 1976.

Van Kampen, N. G., Stochastic differential equations, Phys. Rep., 24 C, 171-228, 1976.

(Received May 30, 1978; revised February 26, 1979; accepted March 13, 1979.) 